\title{
Synchronization in a semiclassical Kuramoto model
}

\author{
Ignacio Hermoso de Mendoza, ${ }^{1}$ Leonardo A. Pachón, ${ }^{2}$ Jesús Gómez-Gardeñes,,${ }^{1,3}$ and David Zueco ${ }^{1,4,5}$ \\ ${ }^{1}$ Departamento de Física de la Materia Condensada, Universidad de Zaragoza, E-50009 Zaragoza, Spain \\ ${ }^{2}$ Grupo de Física Atómica y Molecular, Instituto de Física, Facultad de Ciencias Exactas y Naturales, \\ Universidad de Antioquia UdeA; Calle 70 No. 52-21, Medellín, Colombia \\ ${ }^{3}$ Instituto de Biocomputación y Física de Sistemas Complejos, Universidad de Zaragoza, E-50018 Zaragoza, Spain \\ ${ }^{4}$ Instituto de Ciencia de Materiales de Aragón, CSIC-Universidad de Zaragoza, E-50012 Zaragoza, Spain \\ ${ }^{5}$ Fundación ARAID, Paseo María Agustín 36, E-50004 Zaragoza, Spain
}

(Received 22 August 2013; revised manuscript received 23 June 2014; published 4 November 2014)

\begin{abstract}
Synchronization is a ubiquitous phenomenon occurring in social, biological, and technological systems when the internal rythms of their constituents are adapted to be in unison as a result of their coupling. This natural tendency towards dynamical consensus has spurred a large body of theoretical and experimental research in recent decades. The Kuramoto model constitutes the most studied and paradigmatic framework in which to study synchronization. In particular, it shows how synchronization appears as a phase transition from a dynamically disordered state at some critical value for the coupling strength between the interacting units. The critical properties of the synchronization transition of this model have been widely studied and many variants of its formulations have been considered to address different physical realizations. However, the Kuramoto model has been studied only within the domain of classical dynamics, thus neglecting its applications for the study of quantum synchronization phenomena. Based on a system-bath approach and within the Feynman path-integral formalism, we derive equations for the Kuramoto model by taking into account the first quantum fluctuations. We also analyze its critical properties, the main result being the derivation of the value for the synchronization onset. This critical coupling increases its value as quantumness increases, as a consequence of the possibility of tunneling that quantum fluctuations provide.
\end{abstract}

DOI: 10.1103/PhysRevE.90.052904

PACS number(s): 05.45.Xt

\section{INTRODUCTION}

Synchronization is perhaps the most cross-disciplinary concept emerging from collective behavior [1] as it manifests across many branches of natural and social sciences. Ensembles of neurons, fireflies, or humans are prone to synchronize their internal rhythms when they become sufficiently coupled, producing a macroscopic dynamically coherent state. In all these seemingly unrelated situations, regardless of the precise nature of the coupled units, the interaction drives the system's components to behave homogeneously. Thus, the study of the microscopic rules that drive ensembles towards synchrony has had a long and fruitful history since the seminal observations made by Huygens [2-4].

The mathematical formulation of the early models showing synchronization phenomena dates back to the 1970s when, after some preliminary work by Peskin and Winfree [5], Kuramoto [6] formalized his celebrated model. The Kuramoto model incorporates the minimum dynamical ingredients aimed at capturing a variety of physical phenomena related to the onset of synchronization. In particular, the Kuramoto model links physical concepts such as self-organization, emergence, order in time, and phase transitions and thus is the most paradigmatic framework in which to study synchronization $[5,7,8]$.

Despite the large body of literature devoted to the Kuramoto model and its variants, its study has always been restricted to the classical domain. Initially, given the usual nature (scale) of the systems in which synchronization is typically observed, it seems superfluous thinking of a quantum theory for the Kuramoto model. However, there is not doubt about the fundamental importance of studying quantum fluctuations within the emergence of synchronized states [9-16]. Moreover, the
Kuramoto model has been implemented on circuits and microand nanomechanical structures $[17,18]$, systems that have already met the quantum domain $[19,20]$. At the quantum level, synchronization, understood as the emergence of a coherent behavior from an incoherent situation in the absence of external fields, is reminiscent of phenomena such as Bose-Einstein condensation and has been observed in interacting condensates of quasiparticles [21,22]. Additionally, synchronization has been suggested to occur in ensemble of atoms and enhance the coherence time of the next generation of lasers [23]. Thus, moved by its fundamental and applied importance, in this work we provide the semiclassical version of the Kuramoto model in an attempt to understand the influence that quantumness has on the emergence of synchronized states.

Our work in this paper consists, as stated by Caldeira and Leggett [24], in finding consistent equations that in the classical limit match the Kuramoto model. Our derivation relies on the quantization of open systems in the framework of Feynman's path-integral formalism. We compute quantum corrections to the classical Kuramoto model. We also analyze its critical properties by deriving the critical point from which synchronization appears and determine how quantum fluctuations affect this synchronization transition.

The rest of the paper is organized as follows. In Sec. II we review the main features of the classical model. Section III, which constitutes the main part of our work, presents the semiclassical equations and our numerical results on the synchronization dynamics. In Sec. V we derive the critical value for the synchronization transition. We summarize and discuss our results in Sec. VI. The technical steps for the semiclassical calculations and the critical value are given in the Appendices. 

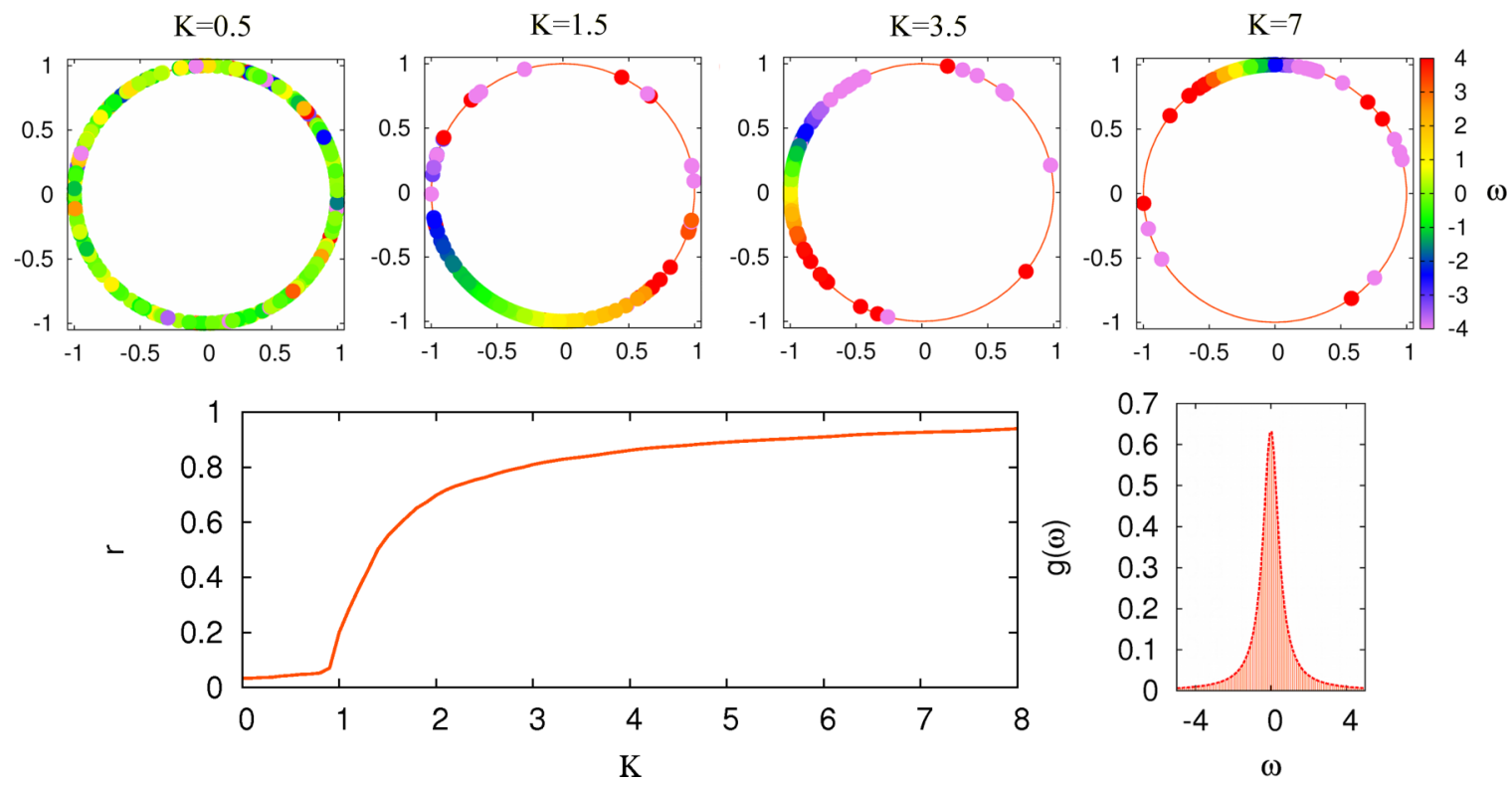

FIG. 1. (Color online) Synchronization in the classical Kuramoto model. Each panel on the top shows the collection of oscillators situated in the unit circle (when each oscillator $j$ is represented as $e^{i \theta_{j}(t)}$ ). The color of each oscillator represents its natural frequency. From left to right we observe how oscillators start to concentrate as the coupling $K$ increases. In the panels below we show the synchronization diagram, i.e., the Kuramoto order parameter $r$ as a function of $K$. It is clear that $K_{c}=1$ as obtained by using the distribution $g(\omega)$ shown in the right panel.

\section{CLASSICAL KURAMOTO MODEL}

The original Kuramoto model [6] considers a collection of $N$ phase oscillators, i.e., it assumes that the characteristic time scale of their amplitudes is much faster than that of the phases. Thus, the dynamical state of the $i$ th unit is described by an angular variable $\theta_{i} \in(0,2 \pi]$ whose time evolution is given by

$$
\dot{\theta}_{i}=\omega_{i}+\frac{K}{N} \sum_{j=1}^{N} \sin \left(\theta_{i}-\theta_{j}\right) .
$$

This equation thus describes a set of weakly coupled phase oscillators whose internal (natural) frequencies $\left\{\omega_{i}\right\}$ are, in principle, different as they are assigned following a frequency distribution $g(\omega)$ that is assumed to be unimodal and even around the mean frequency $\Omega$ of the population $g(\Omega+\omega)=$ $g(\Omega-\omega)$.

In the uncoupled limit $(K=0)$ each element $i$ describes limit-cycle oscillations with characteristic frequency $\omega_{i} . \mathrm{Ku}-$ ramoto showed that by increasing the coupling $K$ the system experiences a transition towards complete synchronization, i.e., a dynamical state in which $\theta_{i}(t)=\theta_{j}(t) \forall i, j$ and $\forall t$. This transition occurs when the coupling strength exceeds a critical value whose exact value is

$$
K_{c}=\frac{2}{\pi g(\Omega)}
$$

To monitor the transition towards synchronization, Kuramoto introduced a complex order parameter

$$
r(t) e^{i \Psi(t)}=\frac{1}{N} \sum_{j=1}^{N} e^{i \theta_{j}(t)} .
$$

The modulus of the above order parameter $r(t) \in[0,1]$ measures the coherence of the collective motion, reaching the value $r=1$ when the system is fully synchronized and $r=0$ for the incoherent solution. On the other hand, the value of $\Psi(t)$ accounts for the average phase of the collective dynamics of the system.

We illustrate in Fig. 1 the synchronization in the Kuramoto model. The top panels show, for different values of the coupling $K$, how the oscillators concentrate as $K$ increases. The bottom panels show the usual synchronization diagram $r(K)$ for which the exact value of $r$ for each $K$ is the result of a time average of $r(t)$ over a large enough time window. In this diagram we can observe that $K_{c}=1$ as a result of using the distribution $g(\omega)$ shown on the right.

Let us note that the all-to-all coupling considered originally by Kuramoto can be trivially generalized to any connectivity structure by introducing the coupling matrix $A_{i j}$ inside the sum in Eq. (1) so that each term $j$ accounting for the interaction between oscillators $i$ and $j$ is assigned a different weight. The latter allows for the study of the synchronization properties of a variety of real-world systems for which interactions between constituents are better described as a complex network [25]. The formalism developed in this work is completely general and valid for any form of $K_{i j}$, thus making possible the extension of the large number of studies of the Kuramoto model in any topology [26] to the semiclassical domain. However, the numerical part of our work will deal with the all-to-all coupling for the sake of comparison with the original Kuramoto work.

\section{QUANTIZATION OF THE KURAMOTO MODEL}

The most important problem when facing the quantization of the Kuramoto model is its non-Hamiltonian character since, as introduced above, Eq. (1) assumes the steady state for the dynamical state of the amplitude of the oscillators. Thus, a question arises as to how we introduce quantum 
fluctuations in the Kuramoto model. One possible choice is to resort to the original microscopic dynamics of amplitude and phases and then identify the underlying Hamiltonian dynamics. However, many different dynamical setups can have the Kuramoto model as their corresponding limiting case of fast amplitude dynamics. Thus, in order to keep the generality of the Kuramoto model, it is desirable not to resort to any specific situation (Hamiltonian) and introduce quantum fluctuations directly.

A similar problem was faced by Caldeira and Leggett in the 1980s [24] when they studied the influence of dissipation in quantum tunneling. In their case, the corresponding classical dynamics dates back to the studies on activation theory by Kramers [27]. Classically, a particle in a potential experiences an energy barrier to surmount, which is typically acquired from thermal fluctuations. On the other hand, a quantum particle finds in tunneling an alternative way to bypass an energy barrier. Caldeira and Leggett were thus interested in quantifying the catalytic effect of tunneling in (effectively) lowering the energy barriers. However, as in the Kuramoto model, Kramers activation theory is based on Langevin equations, i.e., stochastic equations that are not directly obtained from any Lagrangian. Furthermore, most of the reaction rate equations were phenomenological. Therefore, they searched for a consistent way to introduce quantum fluctuations regardless of the microscopic origin of the effective classical evolution. As a by-product their work opened the field of quantum Brownian motion in the most general way.

We take here the same route followed by Caldeira and Leggett to introduce quantum fluctuations in the Kuramoto model. In order to accommodate our dynamical system (1) to the framework provided in [24] we start by writing its corresponding Langevin equation

$$
\dot{\theta}_{i}=-\frac{\partial V}{\partial \theta_{i}}+\xi_{i}
$$

with

$$
V\left(\theta_{1}, \ldots, \theta_{N}\right) \equiv-\sum_{i} \omega_{i} \theta_{i}+\frac{K}{N} \sum_{i, j} \cos \left(\theta_{i}-\theta_{j}\right) .
$$

As usual, $\xi_{i}$ is a Markovian stochastic fluctuating force with $\left\langle\xi_{i}(t)\right\rangle=0$ and $\left\langle\xi_{i}(t) \xi_{j}\left(t^{\prime}\right)\right\rangle=2 \delta_{i j} D \delta\left(t-t^{\prime}\right)$. In the limit $D \rightarrow 0$, Eq. (4) reduces to the Kuramoto model in Eq. (1).

Equation (4) is a Langevin equation in the overdamped limit. It is first order rather than second order in time as the inertia term is neglected. Consequently, the Kuramoto model can be viewed as a set of phases evolving in the overdamped limit. The absence of fluctuations in the limit $D \rightarrow 0$ means that the system of phases is at zero temperature $D \sim T$. Such identification with a Langevin equation has been used already for generalizations of the original Kuramoto model taking into account noise and/or inertial effects [8]. In particular, in [28] it was shown that the critical value $K_{c}$ reads

$$
K_{c}=\frac{2}{\int_{-\infty}^{\infty} d \omega \frac{D}{D^{2}+\omega^{2}} g(\omega)},
$$

which, in the limit $D \rightarrow 0$, recovers the Kuramoto critical coupling (2).
The key point of deriving the Langevin equation (4) corresponding to the Kuramoto model is that it can be obtained from a fully Hamiltonian framework by coupling the system, in our case the coupled phases $\theta_{i}$, to a macroscopic bath or reservoir [27]. In this way, both the damping and fluctuations are seen to be caused by the coupling of the system of phases to the bath. The Hamiltonian description is properly cast in the system-bath approach

$$
H_{\text {tot }}=H_{\text {sys }}+H_{\text {bath }}+H_{\text {int }},
$$

where the bath is an infinite collection of harmonic oscillators with frequencies $\left\{\omega_{\alpha}\right\}$ (note that greek subindexes denote the oscillators in the bath). In the case we are dealing with the total Hamiltonian, which reads

$$
\begin{aligned}
H_{\mathrm{tot}}= & \sum_{i} \frac{\pi_{i}^{2}}{2}+V\left(\theta_{1}, \ldots, \theta_{N}\right) \\
& +\frac{1}{2} \sum_{i, \alpha} P_{i, \alpha}^{2}+\omega_{\alpha}^{2}\left(Q_{i, \alpha}-\lambda_{\alpha} \theta_{i}\right)^{2}
\end{aligned}
$$

where $\left\{\left(\theta_{i}, \pi_{i}\right)\right\}$ and $\left\{\left(Q_{i, \alpha}, P_{i, \alpha}\right)\right\}$ denote the system and bath canonical coordinates, respectively, and $\lambda_{\alpha}$ stands for the coupling constant between bath and system coordinates.

Under well-defined conditions, the equations of motion for the system coordinates derived from the Hamiltonian (8) lead to the previously derived overdamped Langevin equation (4). In particular, one needs to assume (i) thermalized initial conditions for the bath

$$
\begin{gathered}
\left\langle Q_{i, \alpha} Q_{i^{\prime}, \alpha^{\prime}}\right\rangle=\delta_{i, i^{\prime}} \delta_{\alpha, \alpha^{\prime}} k_{B} T / \omega_{\alpha}^{2}, \\
\left\langle P_{i, \alpha} P_{i^{\prime}, \alpha^{\prime}}\right\rangle=\delta_{i, i^{\prime}} \delta_{\alpha, \alpha^{\prime}} k_{B} T
\end{gathered}
$$

(ii) the frequency spectrum of the bath oscillators is flat (this assumption leads to the widely used Ohmic dissipation); and finally (iii) the changes in time of the velocity (acceleration) induced by the energy potentials are far slower than the energy loss induced by the coupling between the system and the bath (this is the situation when the system and the bath are strongly coupled), so we could neglect the inertial term.

\section{Semiclassical equation}

Once we have a Hamiltonian description for the Kuramoto equation (1), we are ready to perform its quantization. First, we associate the phases and their associated momenta with the positions and momenta for the bath by providing them with the canonical commutation rules. The hardest work is to find an effective quantum evolution depending only on phase operators, i.e., the so-called quantum Langevin equation. It turns out that such an operator equation is a differential equation that is nonlocal in time, which makes it extremely difficult to manipulate in general. However, the quantum version of Eq. (4) in the overdamped limit is a $c$-number local differential equation [29-34]. The full derivation for the quantum Langevin equation is based on the path-integral formulation. It is lengthy and rather technical. Let us first present the final result and then a sketch of the derivation. Further details can be found in Appendix A. 
The resulting quantum evolution in the Itô representation reads as follows:

$\dot{\theta}_{i}=-\frac{V_{i}^{\prime}}{F_{i}}+\frac{\Lambda}{F_{i}} \sum_{j}\left(\beta V_{j}^{\prime} V_{i j}^{\prime \prime}-V_{j j i}^{\prime \prime \prime}\right)-\frac{\Lambda}{2 F_{i}} V_{i i i}^{\prime \prime \prime}+\sqrt{\frac{1}{F_{i}}} \cdot \xi_{i}$,

where we have used the compact notation $V_{i, \ldots, k}^{\prime \ldots,} \equiv \partial_{\theta_{i}, \ldots, \theta_{k}} V$, $\xi_{i}$ is an stochastic force with the same statistics as in (4),

$$
F_{i}=e^{-(\Lambda / D) V_{i i}^{\prime \prime}}
$$

and $\Lambda$ is the quantumness parameter

$$
\begin{aligned}
\Lambda & =\frac{2}{m \beta} \sum_{n} \frac{1}{v_{n}^{2}+\gamma v_{n}} \\
& =\frac{\hbar}{m \pi \gamma}\left(\Psi\left[\frac{\hbar \beta \gamma}{2 \pi}\right]-C+\frac{2 \pi}{\hbar \beta \gamma}\right),
\end{aligned}
$$

with $C=0.577 \ldots$ the Euler-Mascheroni constant and $\Psi$ the Digamma function. Note that in the limit $\hbar \beta \gamma \rightarrow 0$, $\Lambda \rightarrow 0$. Making $\Lambda \rightarrow 0$, the quantum Langevin (11) reduces to the classical equation (4). This is a remarkable property. Our result is perturbative in $\beta \Lambda$, giving quantum corrections containing, as a limit, the Kuramoto model. We notice that, being perturbative, $\beta \Lambda$ must be small, which means that our equation is valid at high temperatures and damping. As a drawback of the perturbative character, the model cannot be pushed to the zero-temperature limit. Compared to its classical counterpart $[\beta \Lambda \rightarrow 0$, Eq. (4)], Eq. (11) has a renormalized effective potential (5) (the third term on the right-hand side). Besides, both the diffusion and consequently its noise terms are also modified by the quantum fluctuations (the second and last terms on the right-hand side, respectively).

The noise, because of the $\sqrt{1 / F_{i}}$, is now multiplicative. In the limit $\beta \Lambda \rightarrow 0, F \rightarrow 1$. Hence, in the classical limit the multiplicative noise switches into additive noise. This immediately suggests that the multiplicative nature is related to the underlying quantum stochastic process. Quantum noise depends, undoubtedly, on the state of the system, the dynamics of observables depend on the state the system is in, and therefore quantum noise in a Langevin equation must depend upon the dynamics of the system itself. This explains the multiplicative character of the noise in Eq. (11) at the singlevariable level. This result is consistent with previous works along this line (see, e.g., Ref. [30,32-34]).

\section{Sketch for the derivation of Eq. (11)}

Any Langevin equation, classical or quantum, is an effective evolution of the degrees of freedom for the system of interest. If we start with the total Hamiltonian, the bath degrees of freedom need to be integrated out. In the quantum regime, this means taking the partial trace over the bath Hilbert space. We follow here the program explained in Refs. [29,30]. The steps are as follows. (i) The equilibrium reduced density matrix [35]

$$
\varrho_{\beta}\left(\theta ; \theta^{\prime}\right)=\int d Q W_{\beta}\left(\mathbf{Q}, \theta ; \mathbf{Q}^{\prime}, \theta^{\prime}\right)
$$

is obtained in the overdamped limit. Both $\mathbf{Q}$ and $\theta$ are a shorthand notation to denote the bath and system coordinates.
In such a regime the damping is sufficiently strong to suppress the nondiagonal elements (coherences) of the reduced density matrix, i.e., a regime where $\left\langle\theta_{1}, \ldots, \theta_{N}\left|\varrho_{\beta}\right| \theta_{1}^{\prime}, \ldots, \theta_{N}^{\prime}\right\rangle \sim$ $\prod \delta\left(\theta_{i}-\theta_{i}^{\prime}\right)$. We define

$$
P_{\beta}(\theta):=\varrho_{\beta}(\theta ; \theta) .
$$

As detailed in Appendix A, the reduced density matrix in the overdamped limit can be written as

$$
\begin{aligned}
P_{\beta}(\theta)= & \mathcal{Z}^{-1} \exp \left(-\beta \Lambda \sum_{i} V_{i, i}^{\prime \prime}\right) \\
& \times \exp \left(-\beta V+\frac{1}{2} \beta^{2} \Lambda \sum_{i}\left(V_{i}^{\prime}\right)^{2}\right) .
\end{aligned}
$$

Once the equilibrium density matrix is obtained, (ii) the master equation for the probability distribution $P(q, t)$ [cf. Eq. (15)] is proposed. Taking into account the results for the harmonic oscillator [31] and the single-particle case [29,32], the master equation can be formally written as

$$
\partial_{t} P(\theta ; t)=\partial_{\theta} L P(\theta ; t) .
$$

(iii) The actual master equation takes a Fokker-Planck form. It is obtained by imposing that the equilibrium density distribution $P_{\beta}$ given by Eq. (16) is stationary under (17), $L P_{\beta}(q)=0$. The final result is

$$
\begin{aligned}
\partial_{t} P= & \sum_{i} \frac{\partial}{\partial \theta_{i}}\left\{\left[\frac{V_{i}^{\prime}}{\Gamma F_{i}}-\frac{\beta}{\Gamma F_{i}} \Lambda \sum_{j} V_{j}^{\prime} V_{i, j}^{\prime \prime}+\frac{\Lambda}{\Gamma F_{i}} \sum_{j \neq i} V_{j j i}^{\prime \prime \prime}\right]\right. \\
& \left.+\frac{\partial}{\partial \theta_{i}}\left[\frac{D}{\gamma^{2} F_{i}}\right]\right\} P .
\end{aligned}
$$

Finally, (iv) the Langevin equation (11) is obtained via the equivalence of Fokker-Planck equations, as Eq. (18) and Langevin-type equations [36].

\section{TRANSITION TO SYNCHRONIZATION IN THE SEMICLASSICAL MODEL}

Once we derived the semiclassical version of the Kuramoto equation, it is natural to unveil the effects that quantum fluctuations induce in the transition to synchronization. As introduced previously, to study the synchronization transition one resorts to the order parameter $r$ [introduced in Eq. (11)] that reveals the synchronized state of the system. We solve both the classical Kuramoto model $(\Lambda=0)$ and the quantum one $(\Lambda>0)$ numerically, extracting from the dynamics the stationary value of $r$. Throughout this work, the numerical calculations are performed with $N=10^{3}$ oscillators and the distribution of natural frequencies is Lorentzian:

$$
g\left(\omega ; \omega_{0}, \alpha\right)=\frac{1}{\pi} \frac{\alpha}{\left(\omega-\omega_{0}\right)^{2}+\alpha^{2}},
$$

with $\alpha=0.5$ and centered around $\omega_{0}=0$.

Figure 2(a) shows the typical synchronization diagram, namely, the value of $r$ as a function of the coupling strength $K$. The comparison of the semiclassical (for $\Lambda=0.1$ ) and classical curves $r(K)$ evinces that quantum fluctuations delay the onset of synchronization, i.e., the critical point $K_{c}$ is seen to move to larger values with $\Lambda$. We have also considered the 

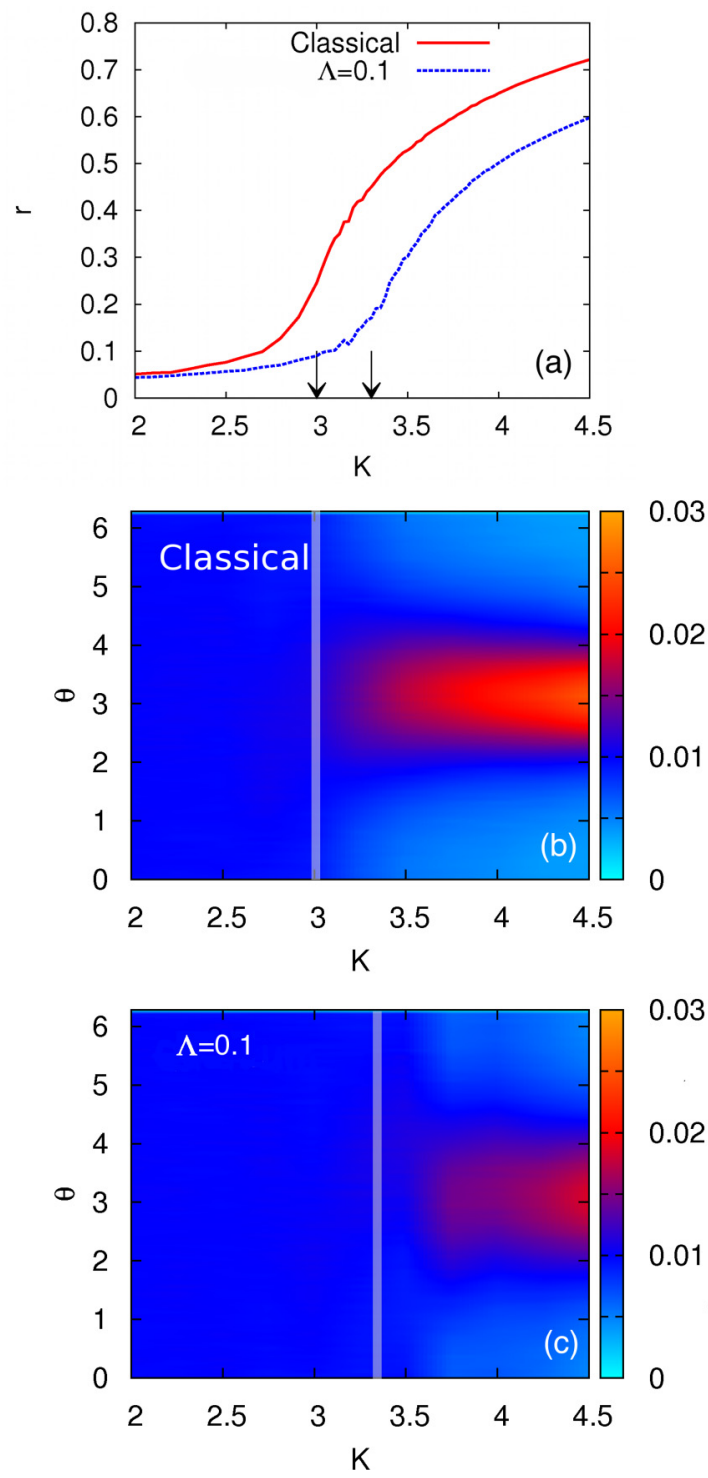

FIG. 2. (Color online) Classical vs semiclassical synchronization transitions. (a) Synchronization diagrams $r(K)$ for the classical $(\Lambda=0)$ and the quantum $(\Lambda=0.1)$ Kuramoto models. In both cases the thermal noise is chosen such that $D=1$. The number of oscillators is $N=10^{3}$ and the distribution of natural frequencies is given in Eq. (19) centered in $\omega_{0}=0$ and $\alpha=0.5$. It is clear that the synchronization onset is delayed as soon as quantumness enters into play. (b) and (c) Probability $P(\theta)$ of finding an oscillator at a given phase $\theta$ as a function of $K$. Note that for each value of $K$, the phases have been equally shifted so that the mean phase is located at $\theta=\pi$. A thick gray line indicates the critical values $K_{c}$ and $K_{c}^{q}$ for classical and quantum dynamics, respectively.

evolution for the distribution of the phases as a function of $K$ to monitor the microscopic distinctive identifying characteristic of the synchronization transition. The evolution of the classical and quantum Kuramoto models is shown in Figs. 2(b) and 2(c), respectively.

To explain the delay in the synchronization onset introduced by quantum fluctuations we resort to the simplest situation: two coupled Kuramoto oscillators. In this case the Kuramoto model (4) consists of just two coupled equations for the (a) Classical

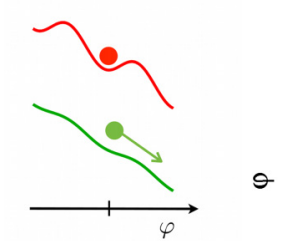

(b) $\Lambda=0.1$
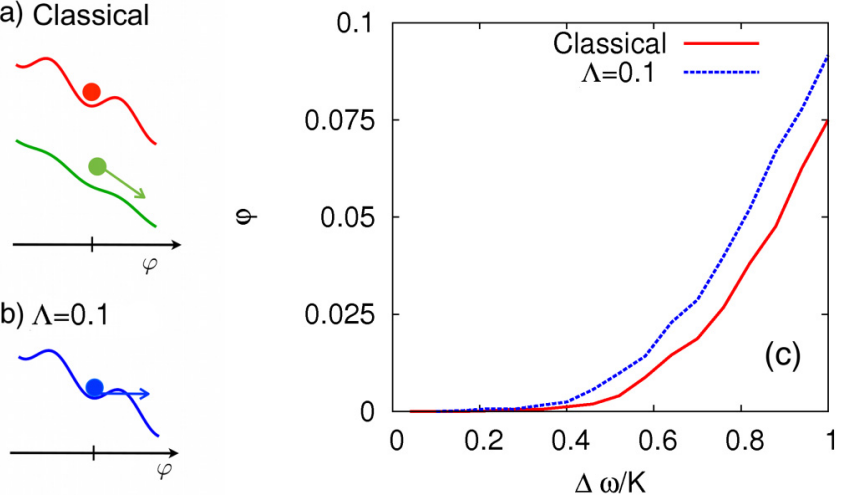

FIG. 3. (Color online) System of two coupled Kuramoto oscillators. (a) Analogy between the system of two coupled oscillators and an overdamped particle in a washboard potential. The two possible regimes are shown: the synchronized state (the particle is at rest $\dot{\varphi}=0$ at a local minimum) and the unsynchronized phase (the particle drifts across the potential). (b) Possibility that tunneling allows one to anticipate the drifting state. (c) Result of the computation of the velocity $\dot{\varphi}$ as a function of $\Delta \omega / K$ for the classical (solid red line) and quantum (dashed blue line) systems.

evolution of $\theta_{1}$ and $\theta_{2}$. By taking the difference of those two equations and introducing as a new variable the phase difference $\varphi:=\theta_{1}-\theta_{2}$, we obtain for its evolution the following equation:

$$
\dot{\varphi}=\Delta \omega-K \sin \varphi+2 D \xi .
$$

This equation describes the evolution of an overdamped particle in a washboard potential (see Fig. 3). With this image in mind, we map the synchronous movement of the two oscillators (defined as a state in which the frequencies of the oscillators are locked: $\dot{\theta}_{1}=\dot{\theta}_{2}$ ) with the resting state of the overdamped particle inside a local minimum of the potential energy $(\dot{\varphi}=0)$. On the other hand, when the two oscillators are not synchronized the particle drifts across the potential $(\dot{\varphi} \neq 0)$. Both situations are shown in Fig. 3 .

The quantum version for the diffusion of an overdamped particle in a periodic potential was previously studied in Ref. [33]. The main result is that the escape rate of the particle, and thus its unlocking mechanism, is enhanced through quantum fluctuations. This effect can be seen as a consequence of the enhancement of the transition probability for energies below the height of the barrier, which is the well-known tunnel effect [37]. In Fig. 3 we show, for both the classical and semiclassical $(\Lambda=0.1)$ systems of two coupled Kuramoto oscillators, the value of $\dot{\varphi}=0$ as a function of the ratio between the difference of the natural frequencies of the two oscillators $|\Delta \omega|$ and the coupling $K$. It is clear that, as stated above, quantum tunneling facilitates the drift or, equivalently, delays the transition to the synchronous state.

\section{ANALYTICAL EXPRESSION FOR THE SYNCHRONIZATION ONSET}

Returning to the original model of $N$ interacting oscillators, we now make an analytical estimation of the value for critical coupling at which the synchronization transition occurs. The 

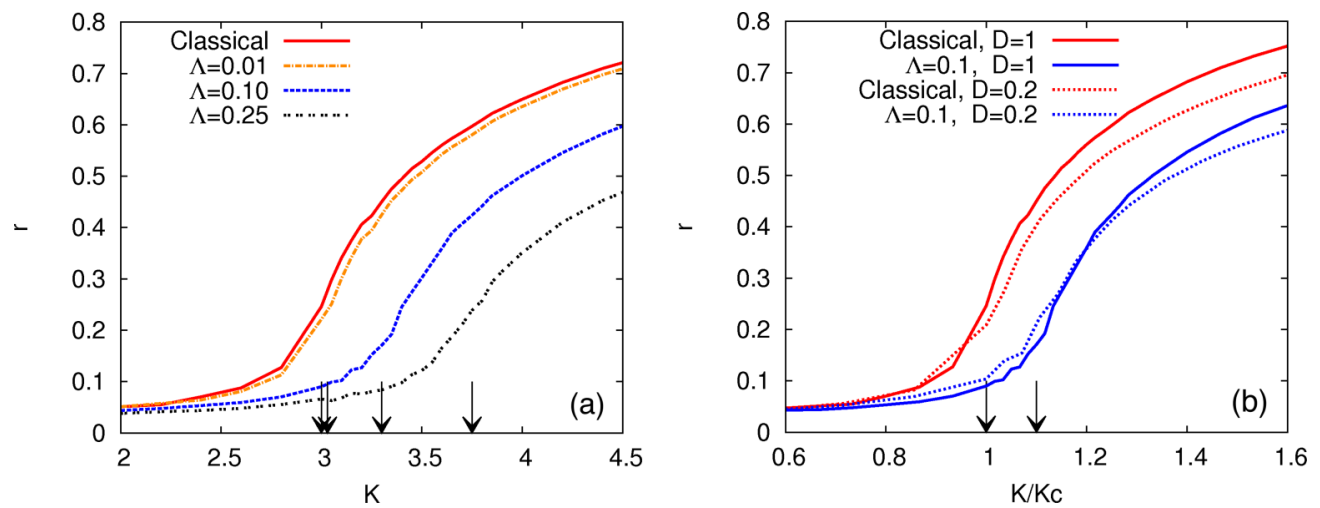

FIG. 4. (Color online) Analysis of the behavior of the critical coupling. (a) Synchronization diagrams $r(K)$ for different values of the degree of quantumness $\Lambda$. Note that the coupling strength has been normalized to its value in the classical $(\Lambda=0)$ limit. The analytical estimation in Eq. (21) is shown by the vertical lines confirming its validity. (b) Plot of $r(K)$ for two different temperatures, corresponding to $D=1$ and 0.2 , for the classical and quantum $(\Lambda=0.1)$ models. Again the analytical estimation is shown by the vertical lines.

procedure is a generalization of the one presented in Ref. [28] and takes advantage of the mean-field description of the Kuramoto model. The derivation (detailed in Appendix B) yields a rather simple equation for the critical coupling:

$$
K_{c}^{q}=(1+\Lambda) K_{c}
$$

with $K_{c}$ the classical critical value shown in Eq. (6).

The above result states that quantum fluctuations act by effectively decreasing the coupling strength with the degree of quantumness $\Lambda$. Returning to the physical image of a particle in a washboard potential, we can consider the effect of the quantum correction by considering the first and third terms on the right-hand side of Eq. (11). In this way, quantum corrections can be cast in the form of an effective potential

$$
V_{\mathrm{eff}}=V+\Lambda V^{\prime \prime}
$$

which in the particular case of the washboard potential reads

$$
V_{\text {eff }}=-\Delta \omega \varphi-(K-\Lambda) \cos \varphi .
$$

This equation makes clear that tunneling is formally reflected by an effective barrier reduction that yields the observed shift to higher values for the critical coupling.

Our analytical estimation for $K_{c}^{q}(\Lambda)$ is plotted in Figs. 2 and 4 (vertical arrows), confirming its validity. To corroborate further the correctness of Eq. (21), we explore the synchronization transition for different values of $\Lambda$ in Fig. 4(a). As expected, the onset of synchronization shifts to higher values as the degree of quantumness increases. Again, the predicted value for $K_{c}^{q}$ is plotted (vertical arrows), corroborating the validity of Eq. (21).

To complete our study, we show in Fig. 4(b) the dependence of the synchronization diagram on the thermal fluctuations $D$ for both the classical and quantum $(\Lambda=0.1)$ cases. In all the curves explored the coupling $K$ is rescaled by the corresponding critical coupling $K_{c}$ in the classical regime. In this way we show for both the classical and quantum cases the robustness of the critical value (21) against temperature changes.

\section{DISCUSSION}

The search for quantum corrections to classical phenomena has been pervasive in physics. Some examples related to our work are the generalization to the quantum domain of chaos [38], dissipation [35], and random walks [39]. Each of these examples has its own difficulties when incorporating quantum fluctuations and unveiling their role. Some of these obstacles are the quantum linearity versus the typical nonlinearity of classical systems and the quantization of the non-Hamiltonian system or phenomenological equations. Overcoming these obstacles provides a consistent quantum description that presents a variety of classical problems and their associated physical phenomena.

Among the most studied phenomena in (classical) complex systems is synchronization. This emergent phenomenon is intriguing as well as beautiful since it ranges from the description of the sympathy of clocks to the neuronal functioning in our brain, thus overcoming the disparate diversity in the spatial and time scales associated with the bunch of systems in which synchronization is observed. However, the concept of synchronization usually associated with the classical domain such as clocks, fireflies, or humans is too macroscopic to think about the need to introduce quantum fluctuations in the description of the associated dynamical models.

Several experimental works have shown that synchronization can be observed in the laboratory within Josepshon junction arrays [40], nanomechanical systems [17], or optomechanical systems [41]. All of these systems share one prominent property: They behave quantum mechanically at sufficiently low temperatures. Therefore, adapting the concept of synchronization to coupled entities within the quantum theory is, apart from an interesting theoretical issue, a necessity imposed by the rapid experimental advances.

A first step consists in taking the most widely used framework for studying synchronization phenomena, the Kuramoto model, and adapting it to the quantum domain. Being a paradigmatic theoretical setup, the quantization of the Kuramoto model avails the theoretical study of quantum synchronization in the widest possible manner. To this end, and to overcome the non-Hamiltonian character of the Kuramoto equations, we have mapped the model to an overdamped 
Langevin equation that has a Hamiltonian description by embedding the system in a bath of oscillators. In this way, the quantization of the Kuramoto model is straightforward and includes its classical counterpart as a limiting case: The quantum version incorporates quantum fluctuations for the phases, while the strength of these quantum corrections is encoded in a single parameter.

The route chosen here must be understood as complementary to the study of particular models of coupled quantum systems. The reason is twofold. First, we aim to be as general as possible. The essence of an emergent phenomenon is its ability to describe very different situations with different microscopic dynamics. This is the goal of the Kuramoto model, as it explains the synchronization without resorting to the specific dynamics. Second, a force brute study of many-body quantum entities is a very difficult task that usually implies the reduction of the system to a few coupled systems. However, the observation of a true synchronization transition demands hundreds or thousands of interacting dynamical systems.

Being general, the results obtained allow us to make general statements about the impact that quantumness has on the synchronization of coupled dynamical units. The most important one is that quantum fluctuations delay the appearance of a synchronized state. The explanation of this effect relies on the fact that in the quantum domain not only do the phases have a different natural frequency but also the fluctuations around the classical trajectories are different depending on those internal rhythms. To illustrate this interpretation we recall the simple case of two coupled Kuramoto oscillators. In this case quantum fluctuations are nothing but thermal-assisted tunneling favoring the phase unlocking. Therefore, the coupling needed to synchronize the two oscillators is higher in the quantum limit.

Finally, we want to point out that in a recent publication the question of synchronization in quantum evolutions was also discussed [11]. Under rather general conditions bounds for the degree of synchronization were found based on the Heisenberg uncertainty principle: The phases, derived as averages of noncommuting operators, cannot take values infinitely close. Instead, in our case, which has focused on the quantum version of the Kuramoto model, we have discussed not the maximum degree of synchronization but the critical onset for the appearance of partially synchronized states. In this case quantumness also limits the emergence of a synchronous state. Therefore, similarly to what happens in quantum chaos, synchronization seems to be a quasiclassical phenomenon [12].

\section{ACKNOWLEDGMENTS}

This work was partially supported by the Spanish MINECO under Projects No. FIS2011-14539-E (EXPLORA program), No. FIS2011-25167, and No. FIS2012-38266-C02-01 and by the Comunidad de Aragón (Grupo FENOL). J.G.-G. was supported by MINECO through the Ramón y Cajal program. L.A.P. was supported by CODI of Universidad de Antioquia under Contract No. E01651 and under the Estrategia de Sostenibilidad 2013-2014 and by COLCIENCIAS of Colombia under Grant No. 111556934912.

\section{APPENDIX A: TECHNICAL DETAILS OF THE SEMICLASSICAL KURAMOTO MODEL}

In this Appendix we provide some technical details to obtain the semiclassical Kuramato model in Eq. (11). As sketched in See III, we need the equilibrium density matrix and to calculate some coefficients in a Fokker-Planck equation and transform the latter to a Langevin-type equation.

\section{Equilibrium density matrix: Path-integral formalism}

Let us compute the equilibrium density matrix. In particular we are interested in the reduced density matrix (at equilibrium)

$$
\varrho_{\beta}=\operatorname{Tr}_{\text {bath }}\left\{W_{\beta}\right\},
$$

where $W_{\beta}$ is the total equilibrium density operator $W_{\beta} \sim$ $e^{-\beta\left(H_{\mathrm{sys}}+H_{\text {bath }}+H_{\text {int }}\right)}$. The equilibrium reduced density matrix can be expressed as [42]

$$
\varrho_{\beta}\left(\boldsymbol{\theta}, \boldsymbol{\theta}^{\prime}\right)=\frac{1}{\mathcal{Z}} \int_{\theta_{1}}^{\theta_{1}^{\prime}} \mathcal{D} \theta_{1} \cdots \int_{\theta_{N}}^{\theta_{N}^{\prime}} \mathcal{D} \theta_{N} \exp \left(-\frac{1}{\hbar} S_{\text {eff }}^{E}[\boldsymbol{\theta}]\right),
$$

with the effective action

$$
\begin{aligned}
S_{\mathrm{eff}}^{E}[\boldsymbol{x}]= & \int_{0}^{\hbar \beta} d \tau\left(\sum_{j} \frac{1}{2} m \dot{\theta}_{j}^{2}+V\left(\theta_{1}, \ldots, \theta_{N}\right)\right) \\
& +\frac{1}{2} \sum_{j} \int_{0}^{\hbar \beta} d \tau \int_{0}^{\hbar \beta} d \sigma K(\tau-\sigma) \theta_{j}(\tau) \theta_{j}(\sigma),
\end{aligned}
$$

which contains the kernel

$$
K(\tau)=\frac{m}{\hbar \beta} \sum_{n}\left|v_{n}\right| \hat{\gamma}\left(\left|v_{n}\right|\right) e^{i v_{n} \tau},
$$

with $v_{n}$ the Matsubara frequencies

$$
v_{n}=\frac{2 \pi n}{\hbar \beta}
$$

and the Laplace transform of the damping kernel given by

$$
\hat{\gamma}(z)=\frac{2}{m} \int_{0}^{\infty} \frac{d \omega}{\pi} \frac{J(\omega)}{\omega} \frac{z}{z^{2}+\omega^{2}} .
$$

\section{Overdamped equilibrium}

Based on previous works [30,31] for the single-particle case, we compute the equilibrium distribution in the overdamped limit. The overdamped dynamics refer to a regime in the parameter space where damping is sufficiently strong to suppress the nondiagonal elements (coherences) of the reduced density matrix, i.e., a regime where $\left\langle\theta_{1}, \ldots, \theta_{N}\left|\varrho_{\beta}\right| \theta_{1}^{\prime}, \ldots, \theta_{N}^{\prime}\right\rangle \sim \prod \delta\left(\theta_{i}-\theta_{i}^{\prime}\right)$. These semiclassical diagonal contributions can be computed perturbatively on the quantum fluctuations.

\section{a. Minimal path}

Let us denote the minimal action path by $x_{i}^{\mathrm{MA}} \equiv \bar{\theta}_{i}$. Besides, since we are interested in the diagonal contributions in the 
imaginary-time path integral in Eq. (A2), this means that the trajectories are taken as

$$
\bar{\theta}_{i}(0)=\bar{\theta}_{i}(\hbar \beta) \equiv \theta_{i},
$$

i.e., periodic trajectories with frequencies $v_{n}$. The minimal action path satisfies the generalized Lagrange equations [43]

$$
m \ddot{\bar{\theta}_{i}}-\frac{\partial V}{\partial \bar{\theta}_{i}}-\int_{0}^{\hbar \beta} d \sigma k(\tau-\sigma) \bar{\theta}_{i}(\sigma)=0 .
$$

The periodic condition in Eq. (A7) suggests the Fourier expansion of $\bar{\theta}_{i}(\tau)$ such that

$$
\bar{\theta}_{i}(\tau)=\sum_{n} \theta_{n, i} e^{i v_{n} \tau}
$$

where the Fourier components satisfy

$$
-v_{n}^{2} \theta_{n, i}+\gamma\left(v_{n}\right) \theta_{n, i}+v_{n, i}=b_{i},
$$

with

$$
v_{n, i}=\int_{0}^{\hbar \beta} d \tau \frac{\partial V}{\partial \theta_{i}} e^{-i v_{n} \tau}
$$

and the inhomogeneous term

$$
b_{i}=\dot{\bar{\theta}}_{i}(\hbar \beta)-\dot{\bar{\theta}}_{i}(0)
$$

which result from the jump and cusp singularities arising from fact that the Fourier series expansion for $\bar{\theta}_{i}(\tau)$ periodically continues the path outside the interval $0 \leqslant \tau \leqslant \hbar \beta$ [43]. Note that terms such as $a_{i}=\bar{\theta}_{i}(\hbar \beta)-\bar{\theta}_{i}(0)$ are, in general, expected. However, since we are interested in the diagonal contributions, they do not contribute to the present case.

At this point, we notice that by setting $n=0$ for $b_{i}$ we obtain

$$
b_{i}=\frac{\hbar \beta}{m} \frac{\partial V}{\partial \theta_{i}} .
$$

In addition, the components $\theta_{n, i}$ with $n \neq 0$,

$$
\theta_{n, i}=\frac{-b_{i}}{v_{n}^{2}+\gamma\left(v_{n}\right)},
$$

are suppressed by dissipation. Hence

$$
\theta_{0, i} \cong \bar{\theta}_{i}(0)+\frac{b_{i}}{\hbar} \Lambda,
$$

where $\Lambda$ measures the quantumness

$$
\begin{aligned}
\Lambda & =\frac{2}{m \beta} \sum_{n} \frac{1}{v_{n}^{2}+\gamma v_{n}} \\
& =\frac{\hbar}{m \pi \gamma}\left(\Psi\left[\frac{\hbar \beta \gamma}{2 \pi}\right]-C+\frac{2 \pi}{\hbar \beta \gamma}\right),
\end{aligned}
$$

with $C=0.577 \ldots$ the Euler-Mascheroni constant. Note that in the limit $\hbar \rightarrow 0, \Lambda \rightarrow 0$, as it must be, thus recovering the classical result.

The contribution of the minimal action can be further simplified by considering that

$$
\frac{1}{2} \int d \tau \dot{\bar{\theta}}_{i}^{2}=\frac{1}{2}\left[\theta_{i}\left(\dot{\bar{\theta}}_{i}(\hbar \beta)-\dot{\bar{\theta}}_{i}(0)\right)-\int d \tau \theta_{i} \ddot{\bar{\theta}}_{i}\right]
$$

together with (A7) and substituting Eq. (A8) for the second term on the right-hand side of Eq. (A17) such that

$$
S=\frac{1}{2} \sum_{i} \theta_{i} b_{i}+\int_{0}^{\hbar \beta} d \tau\left(V-\frac{1}{2} \sum_{i} \bar{\theta}_{i} \partial_{\theta_{i}} V\right) .
$$

By using the relation (A13) and noticing that $\bar{\theta}_{i} \cong \theta_{0, i}\left[\theta_{n, i}\right.$ are suppressed, see Eq. (A14)], we have that $\bar{\theta}_{i}-\theta_{i}=b_{i} \Lambda / \hbar$ [cf. Eq. (A15)]. Hence,

$$
S_{\mathrm{MA}}=\hbar \beta V-\frac{1}{2} \sum_{i} \hbar \beta^{2} \Lambda\left(\partial_{\theta_{i}} V\right)^{2} .
$$

\section{b. Fluctuations around the minimal action path}

We study now the fluctuation around the minimal path

$$
\theta_{i}=\bar{\theta}_{i}+y_{i}
$$

subjected to the boundary conditions

$$
y_{i}(0)=y_{i}(\hbar \beta)=0 \text {. }
$$

Consequently, the correction to the path integral reads

$$
F(q)=\int \mathcal{D} y_{1} \cdots \int \mathcal{D} y_{N} \exp \left(-1 / \hbar \int_{0}^{\hbar \beta} d \tau\langle y|\mathrm{~L}| y\rangle\right)
$$

where we have used an economical notation, in the manner of Dirac, for the quadratic form $\langle y|\mathrm{~L}| y\rangle=\sum L_{i j} y_{i} y_{j}$, with $\mathrm{L}=\left\{\left\{L_{i j}\right\}\right\}$ defined as

$$
\mathrm{L}=-\mathrm{I}\left(m \frac{d^{2}}{d \tau^{2}}+\int_{0}^{\hbar \beta} d \sigma k(\tau-\sigma)\right)+\mathrm{V}^{\prime \prime}
$$

where $I$ is the identity matrix and the second-derivative potential matrix $\mathrm{V}^{\prime \prime}=\left\{\left\{V_{i j}^{\prime \prime}\right\}\right\}$ is defined as

$$
V_{i j}^{\prime \prime}:=\frac{\partial V}{\partial \theta_{i} \partial \theta_{j}} .
$$

We proceed as above and Fourier expand the fluctuations around the minimal path [cf. Eq. (A21)]

$$
y_{i}=\frac{1}{\hbar \beta} \sum_{n} y_{n, i} e^{i v_{n} \tau}
$$

which allows us to effectively replace the boundary condition $y_{i}(0)=0$ in terms of a product of Dirac delta functions $\prod_{i} \delta\left(y_{i}(0)\right)=\prod_{i} \delta\left(1 / \hbar \beta \sum_{n} y_{n, i}\right)$ in the integral expressions above, i.e., by changing

$$
\prod_{i} \delta\left(y_{n, i}\right) \sim \int \prod_{i} d \mu_{i} e^{i / \hbar \beta\left\langle\mu \mid y_{n}\right\rangle},
$$

where $\left\langle\mu \mid y_{n}\right\rangle=\sum_{i} \mu_{i} y_{n, i}$. Therefore,

$$
F(q) \sim \int \prod_{i} d \mu_{i} \prod_{n} \prod_{j} d y_{n, j} e^{i / \hbar \beta\left\langle\mu \mid y_{n}\right\rangle} e^{-1 / \hbar \beta\left\langle y_{n}\left|\mathrm{~A}_{n}\right| y_{n}\right\rangle}
$$

with

$$
\mathrm{A}_{n}=\left|\lambda_{n}+\mathrm{V}^{\prime \prime}, \quad \lambda_{n}=v_{n}^{2}+\right| v_{n} \mid \gamma .
$$


This is a Gaussian integral that can be performed by resorting twice to the formula

$$
\int \prod_{j} d \theta_{j} e^{-\langle x|\mathrm{~A}| x\rangle+\langle b \mid x\rangle}=\sqrt{\frac{\pi^{N}}{\operatorname{det} \mathrm{A}}} e^{-\left\langle b\left|\mathrm{~A}^{-1}\right| b\right\rangle}
$$

so that

$$
F(q) \sim \sqrt{\frac{\prod_{n} \operatorname{det} \mathrm{A}_{n}^{-1}}{\sum_{n} \operatorname{det} \mathrm{A}_{n}^{-1}}}
$$

Up to first order in $1 / \gamma$ we get [cf. Eq. (A28)]

$$
\mathrm{A}_{n}^{-1} \cong \frac{1}{\lambda_{n}} \mathrm{I}-\frac{1}{\lambda_{n}^{2}} \mathrm{~V}^{\prime \prime}
$$

To be consistent, we also need to compute the determinants at first order in $1 / \gamma$ [44],

$$
\operatorname{det} \mathrm{A}_{n}^{-1} \cong \frac{1}{\lambda_{n}^{N}} e^{-\operatorname{Tr}\left(\mathbf{V}^{\prime \prime}\right) / \lambda_{n}} .
$$

Based on all the consideration above, in Appendix B we explicitly present the thermal equilibrium state with first-order corrections in the fluctuations along the semiclassical minimal path results. We further derive the associated Smoluchowski equation. Based on the result obtained in Sec. A, the equilibrium density matrix in the overdamped limit reads [see also Eq. (16)]

$$
\begin{aligned}
P_{\beta}(\theta)= & \frac{1}{\mathcal{Z}} \exp \left(-\beta \Lambda \sum_{i} V_{i, i}^{\prime \prime}\right) \\
& \times \exp \left(-\beta V+\frac{1}{2} \beta^{2} \Lambda \sum_{i}\left(V_{i}^{\prime}\right)^{2}\right),
\end{aligned}
$$

where we have introduced the notation $P_{\beta}(\theta)$. In the overdamped limit only the diagonal elements $\varrho_{\beta}(\theta, \theta)$ matter [cf. Eq. (15)]. We have also introduced the compact notation [see the text below Eq. (11)] $V_{i, \ldots, k}^{\prime \cdots,} \equiv \partial_{\theta_{i}, \ldots, \theta_{k}} V$.

\section{Quantum master equation for the Kuramoto model}

We proceed here as Ankerhold and co-workers did in Refs. [30,31].

\section{a. One-particle master equation}

As an introduction, we consider the one-particle model. In the classical case, the Fokker-Planck equation can be expressed as

$$
\partial_{t} P=\partial_{\theta} L P
$$

where

$$
L=D_{1}(\theta)+\partial_{\theta} D_{2}
$$

with

$$
D_{1}=V^{\prime}=\partial_{\theta} V
$$

and

$$
D_{2}=\frac{D}{\gamma^{2}}=\frac{k_{\beta} T}{m \gamma}=\frac{1}{m \gamma \beta}=\frac{1}{\Gamma \beta} .
$$

Here $\Gamma:=m \gamma$.
Let us switch to the quantum regime. The reduced density matrix for the single-particle case [see (16)] reads

$$
P_{\beta}=\frac{1}{Z} e^{-\beta \Lambda V^{\prime \prime}} \exp \left(-\beta V+\frac{\beta^{2} \Lambda}{2} V^{\prime 2}\right),
$$

where $Z$ is the partition function and

$$
S=-\beta V+\frac{\beta^{2} \Lambda}{2} V^{\prime 2} .
$$

Up to leading order in $\Lambda$,

$$
P_{\beta}=\frac{1}{Z}\left(1-\beta \Lambda V^{\prime \prime}\right) e^{-\beta V}\left(1+\frac{\beta^{2} \Lambda}{2} V^{\prime 2}\right) .
$$

Imposing the consistency condition $\mathcal{L} \varrho_{\beta}=0$ together with the election for $D_{2}$,

$$
D_{2}=\frac{D}{\gamma^{2}}\left(1+\beta \Lambda V^{\prime \prime}\right)=\frac{D}{\gamma^{2} F},
$$

where $F=1-\beta \Lambda V^{\prime \prime}$, we find

$$
D_{1}=\frac{D}{\gamma^{2}} \beta V^{\prime}=\frac{1}{m \gamma} V^{\prime} .
$$

This yields the quantum master equation for the single case in the overdamped limit

$$
\partial_{t} P=\partial_{x}\left\{\frac{1}{m \gamma} V^{\prime}+\partial_{x}\left[\left(\frac{D}{\gamma^{2}}\left(1+\beta \Lambda V^{\prime \prime}\right)\right)\right]\right\} P .
$$

\section{b. The $N$-particle master equation}

The generalization for (A34) and(A35) for the multivariate case reads

$$
L=D_{1, i}(\theta)+\partial_{\theta, i} D_{2, i},
$$

whereas the stationary solution $P_{\beta}$ in Eq. (16) can be rewritten as

$$
P_{\beta}=\frac{1}{Z} e^{-\beta \Lambda \operatorname{Tr}\left(\mathbf{V}^{\prime \prime}\right)} \exp \left(-\beta V+\frac{\beta^{2} \Lambda}{2} \mathbf{V}^{\prime} \cdot \mathbf{V}^{\prime}\right),
$$

where $\operatorname{Tr}\left(\mathrm{V}^{\prime \prime}\right)$ denotes trace of the matrix $\mathrm{V}^{\prime \prime}$. The stationary solution can be always be written as

$$
P_{\beta}(\theta) \equiv \frac{1}{Z} F(\theta) e^{S} .
$$

With the experience gained in the single-particle case, our choice for $F$ and $e^{S}$ will determine the values for $D_{1, i}$ and $D_{2, i}$. If we choose $F=1$ we do not recover the overdamped equation for a quantum harmonic oscillator in the one-particle limit, which is an exact result [31]. On the other hand, we can set, by analogy with the single site case, $F=e^{-\beta \Lambda \operatorname{Tr}\left(\mathrm{V}^{\prime \prime}\right)}$. To recover the uncoupled case, $F$ can be rewritten as $F=\prod_{i} F_{i}$. The actual value for $F_{i}$ must recover the master equation for the harmonic oscillator.

We choose

$$
D_{2, i}=\frac{D}{\gamma^{2} F_{i}}
$$


and impose $P_{\beta}$ [Eq. (A46)] to be the stationary solution

$$
\begin{aligned}
& D_{1, i} P_{\beta}+\partial_{\theta_{i}} D_{2, i} P_{\beta}=D_{1, i} \prod_{j} F_{j} e^{S}+\frac{D}{\gamma^{2}} \partial_{\theta_{i}}\left(\prod_{j \neq i} F_{j}\right) e^{S} \\
& =e^{S}\left[D_{1, i} \prod_{j} F_{j}+\frac{D}{\gamma^{2}}\left(\prod_{j \neq i} F_{j}\right) \partial_{\theta_{i}} S+\frac{D}{\gamma^{2}} \sum_{j \neq i}\left(F_{j, i}^{\prime} \prod_{k \neq j \neq i} F_{k}\right)\right]=0 .
\end{aligned}
$$

Thus,

$$
D_{1, i}=-\frac{D}{\gamma^{2} F_{i}} \partial_{\theta_{i}} S-\sum_{j \neq i} \frac{D}{\gamma^{2} F_{i}} \frac{F_{j, i}^{\prime}}{F_{j}} .
$$

We have already justified the form for $F$, giving

$$
F=\prod_{i} F_{i}=\prod_{i} e^{-\beta \Lambda V_{i i}^{\prime \prime}}
$$

Combining (A47)-(A49), the final form for the master equation is obtained, describing a system of $N$ particles in the Smoluchowski regime

$$
\partial_{t} P=\sum_{i} \frac{\partial}{\partial \theta_{i}}\left\{\left[\frac{V_{i}^{\prime}}{\Gamma F_{i}}-\frac{\beta}{\Gamma F_{i}} \Lambda \sum_{j} \frac{\partial V}{\partial \theta_{j}} \frac{\partial^{2} V}{\partial \theta_{i} \partial \theta_{j}}+\frac{\Lambda}{\Gamma F_{i}} \sum_{j \neq i} V_{j j i}^{\prime \prime \prime}\right]+\frac{\partial}{\partial \theta_{i}}\left[\frac{D}{\gamma^{2} F_{i}}\right]\right\} P .
$$

It is easy to check that setting $N=1$ above the single-particle master equation (A43) is recovered.

\section{c. Langevin equation}

Once we have derived the master equation, we can easily find the associated Langevin equation in the form

$$
\frac{\partial \theta_{i}}{\partial t}=A_{i}(\boldsymbol{\theta}, t)+\sum_{k} B_{i k}(\boldsymbol{\theta}, t) \xi_{k}(t)
$$

following the guidelines explained in Ref. [36] and Chap. 3 in Ref. [46]. Here $\xi_{k}$ is Gaussian $\delta$-correlated white noise with zero mean and variance $2 D$. Following Ref. [36], the Langevin equation is equivalent to the Fokker-Planck-type equation for the probability distribution

$$
\begin{aligned}
\partial_{t} P= & -\sum_{i} \frac{\partial}{\partial \theta_{i}}\left\{\left[A_{i}+D \sum_{j k} B_{j k} \frac{\partial B_{i k}}{\partial \theta_{j}}\right] P\right\} \\
& +D \sum_{i j} \frac{\partial^{2}}{\partial \theta_{i} \partial \theta_{j}}\left\{\left[\sum_{k} B_{i k} B_{j k}\right] P\right\} .
\end{aligned}
$$

The coefficients $A_{i}$ and $B_{i j}$ can be identified by comparing (A50) and (A52). For the concrete case of the Kuramoto potential (5), we finally end up with the semiclassical Kuramoto model in Eq. (11).

\section{APPENDIX B: CRITICAL COUPLING VALUE}

We generalize here the work presented in Ref. [28] to the quantum domain.

\section{Periodicity and self-consistency of the master equation}

The order parameter $r$ is given by

$$
r e^{i\left(\omega_{0} t+\phi_{0}\right)}=\frac{1}{N} \sum_{j=1}^{N} e^{i \phi_{j}}
$$

The Kuramoto potential $V(5)$ in a mean-field approximation reads

$$
V=-\omega \psi-K r \cos \psi .
$$

Neither $V$ nor the stationary solution (16) is $2 \pi$ periodic. We have to find a periodic stationary solution. Following a procedure similar to the one performed by Risken (see Ref. [46], pp. 98 and 287-288), we derive the periodic stationary solution

$$
P(\psi ; \omega)=e^{-\beta V_{\text {eff }}} P(0 ; \omega)\left[1+\frac{\left(e^{-2 \beta \pi \omega}-1\right) \int_{0}^{\psi} d \phi e^{\beta V_{\text {eff }}}}{\int_{0}^{2 \pi} d \phi e^{\beta V_{\text {eff }}}}\right],
$$

with $V_{\text {eff }}=V-\frac{1}{2} \beta \Lambda V^{\prime 2}+\Lambda V^{\prime \prime}$. In the classical limit $\Lambda \rightarrow$ $0, V_{\text {eff }} \rightarrow V$, recovering the classical periodic stationary solution derived by Sakaguchi [28]. It is not hard to check that the $2 \pi$-periodic distribution (B3) is also a stationary solution for (A50).

\section{Critical value}

We follow Sakaguchi [28] to find the critical coupling strength $K_{c}^{q}$. The order parameter $r$ can be expressed in terms of $\psi$ as

$$
r=\int_{-\infty}^{\infty} d \omega g(\omega) \int_{0}^{2 \pi} d \psi n(\psi ; \omega) \exp (i \psi) .
$$


Replacing (B3) above, we have a self-consistent equation for $r$. On the right-hand side of (B4), the imaginary part is always zero because $g(\omega)$ is symmetric around $\omega=0$. The real part is expanded in powers of $K r / D$,

$$
r=K r\left[\int_{-\infty}^{\infty} d \omega g(\omega) \frac{\pi \omega / D\left[1+\Lambda\left(\omega^{2} / D^{2}-1\right)\right][1+\operatorname{coth}(\pi \omega / D)]}{\omega^{2} / D^{2}+1}\right]+O\left((K r / D)^{2}\right) .
$$

Assuming a peaked $g(\omega)$ distribution, we also expand around $\omega=0$, obtaining

$$
r=K r\left[\int_{-\infty}^{\infty} d \omega g(\omega) \frac{(1-\Lambda)(1+\pi \omega / D)}{\left(\omega^{2} / D^{2}+1\right)}\right]+O\left((K r / D)^{2}\right)
$$

Since $g(\omega)$ is an even function, the linear term $\pi \omega / D$ does not contribute to the integral. Finally, the critical coupling strength, as a function of the temperature, is obtained from (B6),

$$
K_{c}^{q}(\beta)=\frac{2}{(1-\Lambda) \int_{-\infty}^{\infty} d \omega g(\omega) \frac{D^{2}}{\omega^{2}+D^{2}}} .
$$

As $K$ increases, a nontrivial solution branches off the trivial solution $r=0$ at $K=K_{c}$. This solution reduces to the classical one [5,28] when $\Lambda=0$ at the classical critical coupling strength $K_{c}^{c}$. A simple relation between the classical and the quantum critical values can be obtained

$$
K_{c}^{q}\left(K_{c}^{c} ; \Lambda\right)=\frac{K_{c}^{c}}{1-\Lambda} .
$$

[1] S. H. Strogatz, Sync (Penguin, New York, 2004).

[2] A. Pikovsky, Synchronization: A Universal Concept in Nonlinear Sciences (Cambridge University Press, Cambridge, 2003).

[3] S. C. Manrubia, A. S. Mikhailov, and D. H. Zanette, Emergence of Dynamical Order (World Scientific, Singapore, 2004).

[4] S. Boccaletti, The Synchronized Dynamics of Complex Systems (Elsevier, Amsterdam, 2008).

[5] S. H. Strogatz, Physica D 143, 1 (2000).

[6] Y. Kuramoto, in International Symposium on Mathematical Problems in Theoretical Physics, edited by H. Araki, Lecture Notes in Physics Vol. 39 (Springer, Berlin, 1975), pp. 420-422.

[7] S. Nadis, Nature (London) 421, 780 (2003).

[8] L. L. Bonilla, C. J. P. Vicente, and F. Ritort, Rev. Mod. Phys. 77, 137 (2005).

[9] I. Goychuk, J. Casado-Pascual, M. Morillo, J. Lehmann, and P. Hänggi, Phys. Rev. Lett. 97, 210601 (2006).

[10] O. V. Zhirov and D. L. Shepelyansky, Phys. Rev. Lett. 100, 014101 (2008).

[11] A. Mari, A. Farace, N. Didier, V. Giovannetti, and R. Fazio, Phys. Rev. Lett. 111, 103605 (2013).

[12] Y. Liu, F. Piéchon, and J.-N. Fuchs, Europhys. Lett. 103, 17007 (2013).

[13] G. L. Giorgi, F. Galve, G. Manzano, P. Colet, and R. Zambrini, Phys. Rev. A 85, 052101 (2012).

[14] G. Manzano, F. Galve, G. L. Giorgi, E. Hernández-García, and R. Zambrini, Sci. Rep. 3, 1439 (2013).

[15] T. E. Lee and H. R. Sadeghpour, Phys. Rev. Lett. 111, 234101 (2013).

[16] T. E. Lee, C.-K. Chan, and S. Wang, Phys. Rev. E 89, 022913 (2014).

[17] M. H. Matheny, M. Grau, L. G. Villanueva, R. B. Karabalin, M. C. Cross, and M. L. Roukes, Phys. Rev. Lett. 112, 014101 (2014).

[18] K. L. Turner, S. A. Miller, G. P. Hartwell, N. C. MacDonald, S. H. Strogatz, and S. Adams, Nature (London) 396, 149 (1998).

[19] M. D. LaHaye, O. Buu, B. Camarota, and K. C. Schwab, Science 304, 74 (2004).
[20] A. D. O'Connell, M. Hofheinz, M. Ansmann, R. C. Bialczak, M. Lenander, E. Lucero, M. Neeley, D. Sank, H. Wang, M. Weides, J. Wenner, J. M. Martinis, and A. N. Cleland, Nature (London) 464, 697 (2010).

[21] A. Baas, K. G. Lagoudakis, M. Richard, R. André, L. S. Dang, and B. Deveaud-Plédran, Phys. Rev. Lett. 100, 170401 (2008).

[22] P. Cristofolini, A. Dreismann, G. Christmann, G. Franchetti, N. G. Berloff, P. Tsotsis, Z. Hatzopoulos, P. G. Savvidis, and J. J. Baumberg, Phys. Rev. Lett. 110, 186403 (2013).

[23] M. Xu, D. A. Tieri, E. C. Fine, J. K. Thompson, and M. J. Holland, arXiv:1307.5891.

[24] A. O. Caldeira and A. J. Leggett, Ann. Phys. (N.Y.) 149, 374 (1983).

[25] S. Boccaletti, V. Latora, Y. Moreno, M. Chavez, and D. U. Hwang, Phys. Rep. 424, 175 (2006).

[26] A. Arenas, A. Díaz-Guilera, J. Kurths, Y. Moreno, and C. Zhou, Phys. Rep. 469, 93 (2008).

[27] P. Hänggi, P. Talkner, and M. Borkovec, Rev. Mod. Phys. 62, 251 (1990).

[28] H. Sakaguchi, Prog. Theor. Phys. 79, 39 (1988).

[29] J. Ankerhold, P. Pechukas, and H. Grabert, Phys. Rev. Lett. 87, 086802 (2001).

[30] J. Ankerhold, H. Grabert, and P. Pechukas, Chaos 15, 026106 (2005).

[31] S. A. Maier and J. Ankerhold, Phys. Rev. E 81, 021107 (2010).

[32] L. Machura, M. Kostur, P. Hänggi, P. Talkner, and J. Łuczka, Phys. Rev. E 70, 031107 (2004).

[33] J. Łuczka, R. Rudnicki, and P. Hänggi, Physica A 351, 60 (2005).

[34] L. Machura, M. Kostur, P. Talkner, J. Łuczka, and P. Hänggi, Physical Review E 73, 031105 (2006).

[35] U. Weiss, Quantum Dissipative Systems, 2nd ed. (World Scientific, Singapore, 2012).

[36] J. L. Garcia-Palacios, arXiv:cond-mat/0701242.

[37] J. L. Garcia-Palacios and D. Zueco, J. Phys. A: Math. Gen. 37, 10735 (2004). 
[38] E. Ott, Chaos in Dynamical Systems, 2nd ed. (Cambridge University Press, Cambridge, 2002).

[39] E. Sánchez-Burillo, J. Duch, J. Gómez-Gardeñes, and D. Zueco, Sci. Rep. 2, 605 (2012).

[40] K. Wiesenfeld, P. Colet, and S. H. Strogatz, Phys. Rev. E 57, 1563 (1998).

[41] G. Heinrich, M. Ludwig, J. Qian, B. Kubala, and F. Marquardt, Phys. Rev. Lett. 107, 043603 (2011).

[42] G.-L. Ingold, in Coherent Evolution in Noisy Environments, edited by A. Buchleitner and K. Hornberger, Lecture Notes in Physics Vol. 611 (Springer, Berlin, Heidelberg, 2002), pp. 1-53.
[43] H. Grabert, P. Schramm, and G. L. Ingold, Phys. Rep. 168, 115 (1988).

[44] Here we have made use of the results derived in Ref. [45]. In particular, we define $M=D+O$, which can be reexpressed as $M=D(I+A)$, with $A=D^{-1} O$. The determinant of $M$ reads $\operatorname{det} \mathrm{M}=\operatorname{det} \mathrm{D} e^{\operatorname{Tr}[\ln (\mathrm{I}+\mathrm{A})]}$, with $\ln (\mathrm{I}+\mathrm{A})=\mathrm{A}-\frac{1}{2} \mathrm{~A}^{2}+\frac{1}{3} \mathrm{~A}^{3}-\cdots$ if $\rho(\mathrm{A})<1$.

[45] I. C. F. Ipsen and D. J. Lee, arXiv:1105.0437.

[46] H. Risken, The Fokker-Planck Equation: Methods of Solution and Applications, Lecture Notes in Mathematics (Springer Science \& Business Media, Berlin, 1996). 